\title{
Top-Down RSFQ Logic Design Based on a Binary Decision Diagram
}

\author{
Nobuyuki Yoshikawa and Junichi Koshiyama
}

\begin{abstract}
We have proposed a top-down design methodology for RSFQ logic circuits using a binary decision diagram (BDD). The BDD is a way to represent a logical function by a directed graph, which consists of binary switches having one input and two outputs. The important features of the BDD RSFQ logic circuits are a small number of primitives, dual rail and non-clocked logic style, and a small gate count. We have constructed a cell library for the BDD RSFQ logic design, which is composed of five square basic cells. Any logic function can be constructed by simply connecting the library cells. CAD tools for the logic level simulation, the circuit simulation and a layout view extraction have been developed to carry out the top-down RSFQ logic design on the Cadence CAD environment. A design flow of the RSFQ full adder is demonstrated to show the potential of the top-down design methodology for the design of large-scale RSFQ integrated circuits.
\end{abstract}

Index Terms-binary decision diagram, CAD, design methodology, RSFQ, superconducting electronics.

\section{INTRODUCTION}

$\mathrm{R}$ apid single flux quantum (RSFQ) integrated circuits are being developed widely because of their potentially high performance with high clock frequency and extremely low power consumption [1]. Some degree of the integration scale of RSFQ digital circuits containing several thousands of Josephson junctions have been successfully implemented and their high performance has been confirmed [2]. For further increase of the integration scale, however, the development of design methodologies as well as CAD tools becomes critical. Recent progress in this field is surveyed in [3].

At the present time a wide range of CAD tools are available to design semiconductor integrated circuits which include multi-million transistors. However straightforward utilization of the semiconductor CAD tools for the design of RSFQ integrated circuits is difficult due to the following reasons:

(i) The RSFQ circuits use voltage pulses to represent logical bit information.

(ii) Interaction between the RSFQ gates is considerable, so that the propagation delay and the bias margin are strongly affected by their neighbors.

Manuscript received September 18, 2000.

Part of this work was performed through Special Coordination Funds for promoting Science and Technology of the Science and Technology Agency of the Japanese government.

N. Yoshikawa, and J. Koshiyama are with Faculty of Engineering, Yokohama National University, Yokohama 240-8501, Japan (telephone: 81-45-339-4259, e-mail: yoshi@yoshilab.dnj.ynu.ac.jp). (iii) Josephson transmission lines (JTLs) are required for the interconnection, which costs considerably large area and large propagation delay. Though microstriplines are possible to use for the long interconnection, they are still costly for the short interconnection because they need additional impedance matching circuits.

Especially, (ii) makes the cell-based design approach difficult and (iii) is an obstacle to realize the automated placing and routing tools. We cannot utilize the semiconductor logic synthesis tools as they are for the RSFQ circuit systems because their circuit styles and the figure of merit for the optimization differ too much.

We have proposed a cell-based design methodology for the RSFQ circuit design based on a binary decision diagram (BDD) [4], [5]. We have demonstrated that the circuit complexity and the propagation delay are improved by using the BDD and the implementation effort can be dramatically reduced by using the cell-based layout approach. Now, we are developing a top-down design methodology for the BDD RSFQ circuits and preparing a CAD environment to automate the design process. In this paper we will introduce a design flow of a BDD RSFQ full adder to show the potentiality of our top-down design methodology.

\section{Cell-Based Design ApProach Using Binary Decision DIAGRAM}

\section{A. BDD and BDD RSFQ Logic}

The BDD is a way to represent the logical function by a directed graph, which is extensively used for the design of the semiconductor pass-transistor logic circuits [6], [7]. Fig. la is an example of the BDD representing the logic function $f=x_{1} x_{2}+\bar{x}_{3}$. It consists of binary switches having one input (root) and two outputs (branch 0 and branch 1). When a messenger is applied to the root, it will be switched into one of the two branches depending on the internal state of the binary switch. In the case of Fig. 1a, if we assume $x_{1}=1, x_{2}=0$, $x_{3}=1$, the messenger propagates along $x_{1}, x_{2}$ and $x_{3}$, then reaches to the box denoted as " 0 ". This value corresponds to a solution of the logic function.

The BDD RSFQ logic circuits can be implemented by replacing the messenger by the SFQ pulse and the binary switches by the RSFQ $D_{2}$ flip-flop [8] as shown in Fig. 1b. In this circuit, the internal state is set to " 1 " ("0"), which corresponds to the clockwise (counter clockwise) current in the storage loop formed by $\mathrm{J}_{2}, \mathrm{~J}_{1}, \mathrm{~L}_{\mathrm{s}}, \mathrm{J}_{6}$ and $\mathrm{J}_{5}$, by inputting an SFQ pulse to the "set1" ("set0"). Then, when an SFQ pulse is 


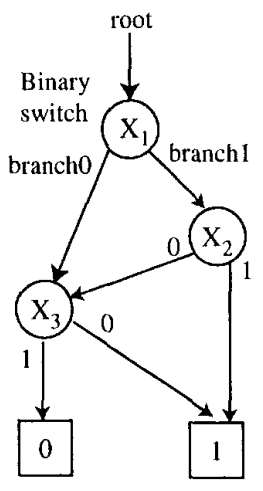

(a)

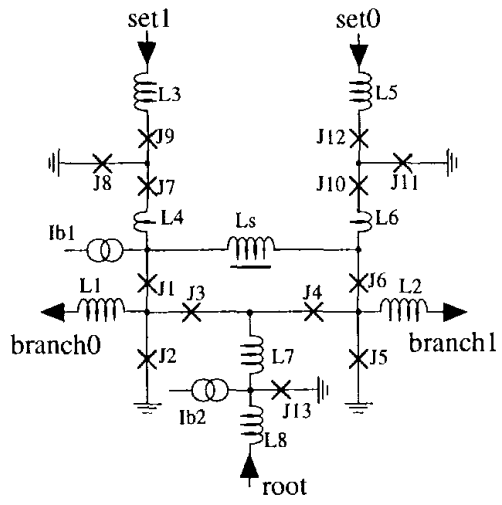

(b)
Fig. 1. (a) A binary decision diagram (BDD). The figure represents a logical function $f=x_{1} x_{2}+\bar{x}_{3}$. (b) Implementation of the BDD binary switch by the $\mathrm{D}_{2}$ flip-flop.

applied to the "root", it goes out through "branch 1" ("branch $0^{\prime \prime}$.

The BDD RSFQ circuits have the following advantages:

(i) Any logic function can be implemented by the combination of only one kind of primitive gates, i.e. $\mathrm{D}_{2}$ flip-flop.

(ii) The circuits are dual rail logic and don't need a global clock.

(iii) Circuit regularity and modularity are very high.

All these features of the BDD RSFQ circuits are suitable for introducing the cell-based design methodology.

\section{B. Cell-Based Design Approach}

We have made a standard cell library for the BDD RSFQ circuits [5]. Symbols of the basic cells in the cell library are shown in Fig. 2. The basic cells consist of four kinds of square cells: one cell having a functionality of the binary switch (Bina) and four cells for interconnection (Line, Cross, Fork and Join). The circuits are specially designed and optimized for the cell library. For instance, the Bina has two additional output terminals, $\mathrm{p} 0$ and $\mathrm{pl}$, to pass the set signal ( $\mathrm{s} 0$ and $\mathrm{s} 1$ ) to its neighbor. All basic cells are optimized not to influence each other, so that the propagation delay and the bias margin are not affected by any combination of interconnections. On the layout level, all cells have identical heights and widths except that the Bina is twice the size of the other cells. Because all positions of input and output terminals are at the same relative position in each cell, any logic function can be fabricated by simply connecting the basic cells.

\section{TOP-DOWN DESIGN FLOW OF THE BDD RSFQ CIRCUITS}

Fig. 3 shows the top-down design flow of the BDD RSFQ logic circuits. CAD tools in this design flow have been integrated into the Cadence $\mathrm{CAD}$ environment. In this section we will show their details through a full adder design as an example.

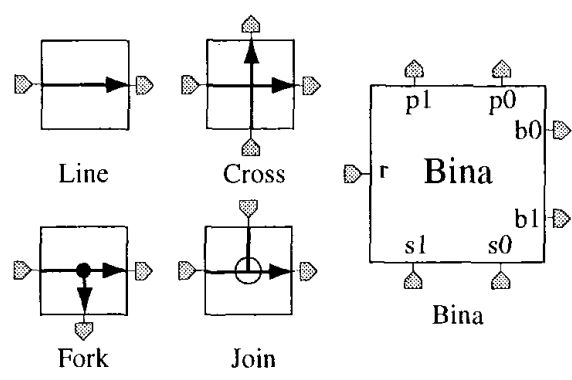

Fig. 2. Symbols of the basic cells in the BDD RSFQ standard cell library:

\section{A. Logic Synthesis by BDD.}

The first step in the top-down design flow is the logic synthesis using the BDD. In this step the desired logic function is synthesized and expressed by the BDD. The logic synthesis method by the BDD is described in [7]. Let's consider a 1-bit full adder here. The binary tree representation of the sum $s=a b c_{\mathrm{i}}+a \bar{b} \bar{c}_{\mathrm{i}}+\bar{a} b \bar{c}_{\mathrm{i}}+\bar{a} \bar{b} c_{\mathrm{i}}$ and the carry output $c_{0}=a b+b c_{\mathrm{i}}+a c_{\mathrm{i}}$ can be generated from the truth table as shown in Fig. $4 \mathbf{a}$ and $\mathbf{b}$, where $a$ and $b$ are the adder inputs and $c_{\mathrm{i}}$ is the carry input.

There are two operations to reduce the size of the binary tree. The first operation eliminates the node whose two outgoing passes point to the same node as shown in Fig. 4c. The node $\mathrm{A}$ is removed in the case of the figure. The second

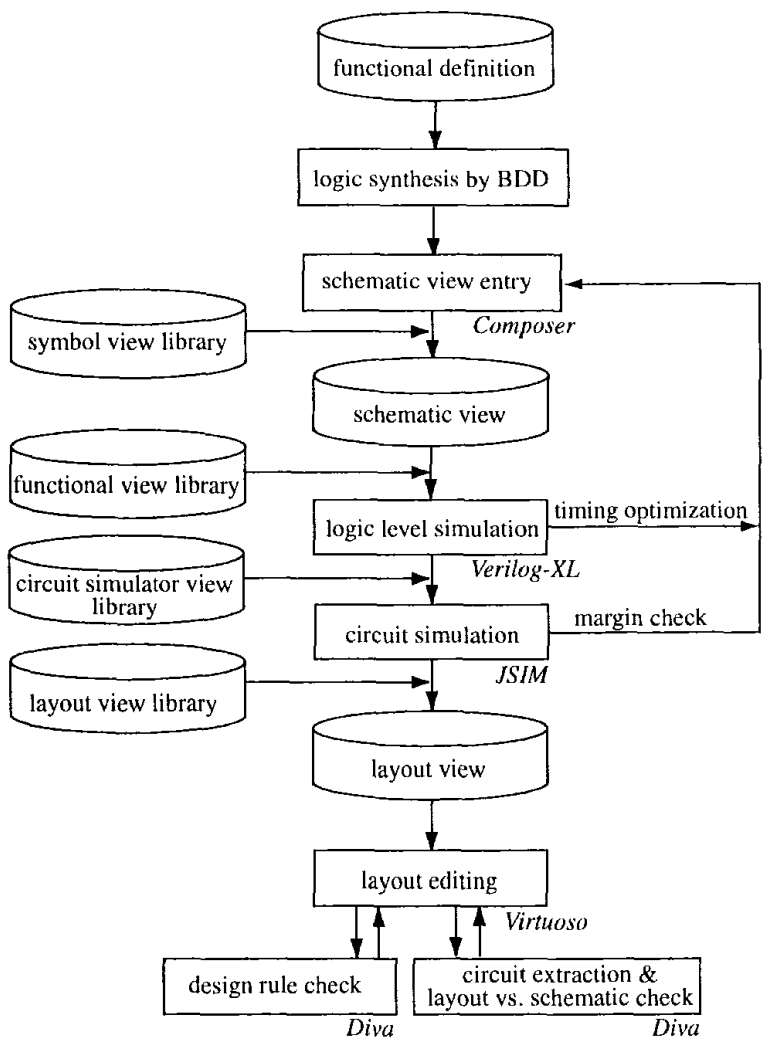

Fig. 3. Top-down design flow of the BDD RSFQ logic circuits. 
operation merges two nodes where corresponding outgoing passes from the two nodes point to the same nodes as shown in Fig. 4d. The node A1 and A2 are merged in the figure. By applying these two operations successively, we can derive the BDD as shown in Fig. 4e and $\mathrm{f}$. We can also merge the two BDDs furthermore by combining the common graphs in $s$ and $c_{\mathrm{i}}$ as shown in Fig. $4 \mathrm{~g}$.

\section{B. Schematic View Entry}

The second step in the design flow is the schematic view entry. In this step a designer makes a circuit schematic view by placing the tile-shaped symbols according to the BDD on the CAD tool using the symbol view library. A schematic view of the 1-bit full adder is shown in Fig. 5. It should be noted that the schematic view reflects the position and the area as well as the logical connection of the circuits. Therefore a designer has to consider not only timing but also circuit layout in this design step.

\section{Logic Level Simulation}

In the next step a designer checks the functionality of their circuits and optimizes the timing by the logic level simulation. The key point of timing design in the BDD RSFQ logic circuits is the relative timing between the pulses arriving at
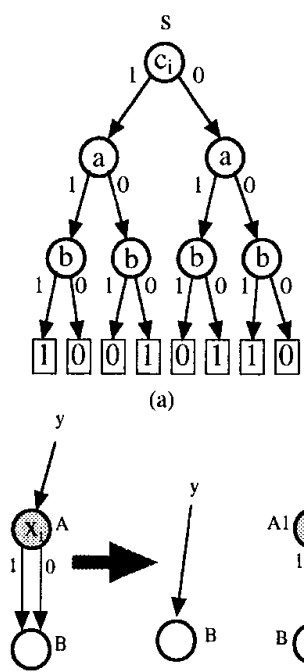

(c)

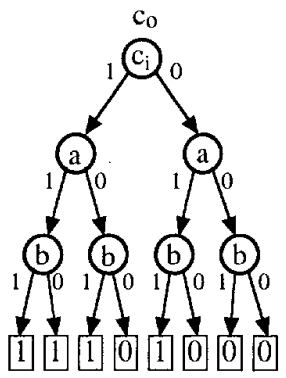

(b)

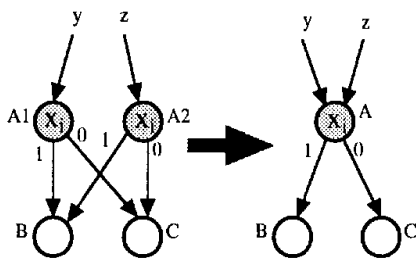

(d)

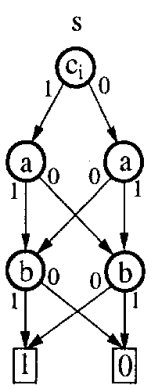

(e)

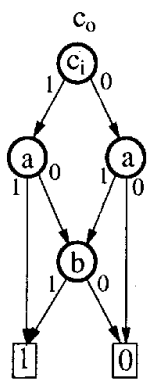

(f)

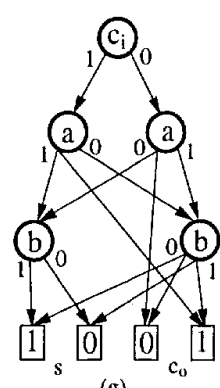

(g)
Fig. 4. Logic synthesis by the BDD. (a), (b) Representation of a Boolean function of a full adder by a logic binary tree. (c), (d) Two basic operations to reduce a logic binary tree. (e), (f) BDD expressions of the sum and the carry output. (g) Merged BDD. the cell terminals that set the state of the Bina ( $\mathrm{s} 0$ and $\mathrm{s} 1$ ) and the pulses arriving at the "root" terminal. The internal state of the Bina has to be set before the arrival of the "root" pulse.

Results of the logic level simulation of a 1-bit full adder are shown in Fig. 6. If there is a timing violation, a designer goes back to the schematic view entry level and modifies the circuit structure. We prepared several kinds of Line cells in order to tune the timing: one cell includes the several JTL stages to increase the delay, another cell includes one JTL but occupies twice the size of the basic cell area to reduce the delay (see rectangular cells in Fig. 5). The HDL description for the logic simulation is generated automatically from the schematic view using the functional view library in the CAD environment. We use the Verilog-XL for the logic simulation. The way to make the timing models of the RSFQ gates and to implement them on the Verilog-HDL is similar to [9]. In our Verilog-HDL environment, however, a designer can calculate the bias voltage dependence of the logic timing.

\section{Circuits Simulation}

The next step is a circuit simulation using JSIM [10]. In this step the timing and the bias margin of the circuit are checked

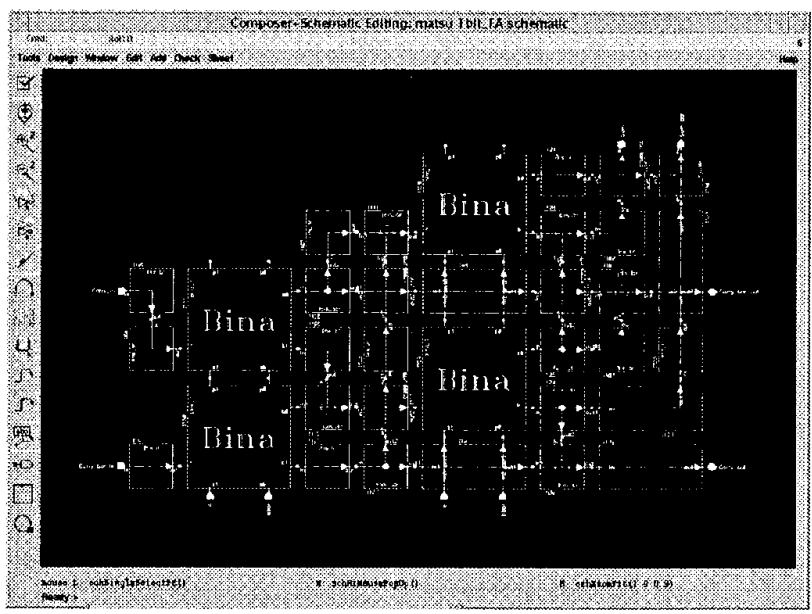

Fig. 5. A schematic view of the BDD RSFQ 1-bit full adder.

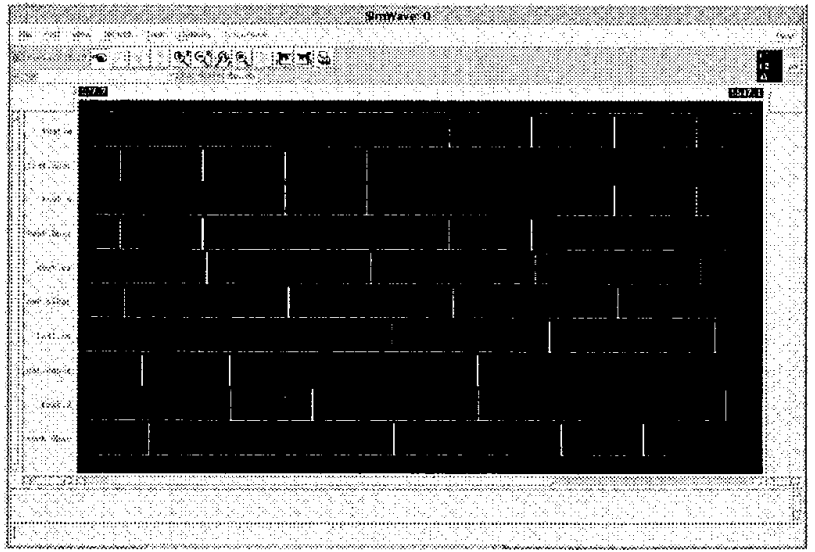

Fig. 6. Logic simulations of the BDD RSFQ 1-bit full adder by Verilog-XL. 


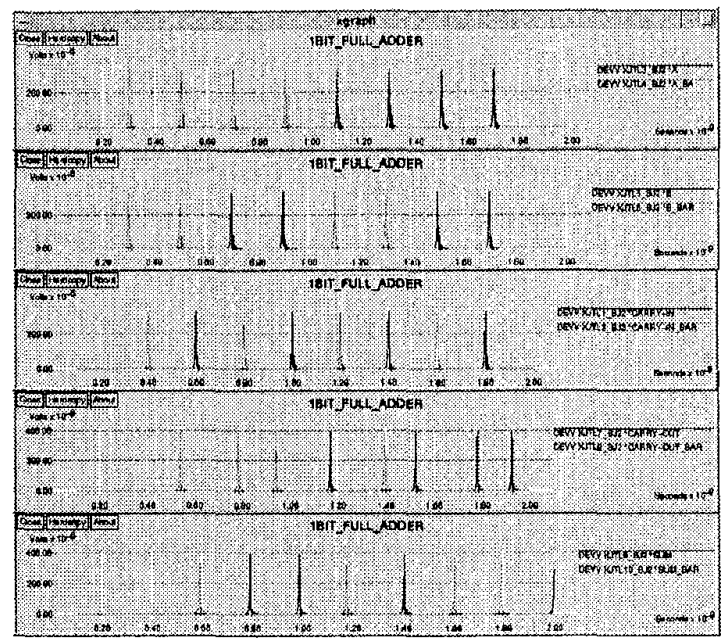

Fig. 7. Circuit simulations of the BDD RSFQ 1-bit full adder by the JSIM.

TABLE I

COMPARISON OF SIMULATION RESULTS OF 1-BIT FULl ADDER.

\begin{tabular}{|c|c|c|c|c|c|c|c|c|}
\hline \multirow[b]{2}{*}{ a } & \multirow[b]{2}{*}{$\mathrm{b}$} & \multirow[b]{2}{*}{$c_{i}$} & \multirow[b]{2}{*}{$\mathrm{c}_{\mathrm{o}}$} & \multirow[b]{2}{*}{$\mathrm{s}$} & \multicolumn{2}{|c|}{ delay_Carry[ps] } & \multicolumn{2}{|c|}{ delay_Sum[ps] } \\
\hline & & & & & Verilog & Jsim & Verilog & Jsim \\
\hline 0 & 0 & 0 & 0 & 0 & 140 & 140 & 191 & 191 \\
\hline 0 & 0 & 1 & 0 & 1 & 182 & 182 & 191 & 191 \\
\hline 0 & 1 & 0 & 0 & 1 & 140 & 140 & 186 & 186 \\
\hline 0 & 1 & 1 & 1 & 0 & 167 & 170 & 176 & 180 \\
\hline 1 & 0 & 0 & 0 & 1 & 195 & 200 & 203 & 200 \\
\hline 1 & 0 & 1 & 1 & 0 & 126 & 129 & 205 & 208 \\
\hline 1 & 1 & 0 & 1 & 0 & 180 & 186 & 190 & 186 \\
\hline 1 & 1 & 1 & 1 & 1 & 126 & 129 & 200 & 200 \\
\hline
\end{tabular}

precisely. We plan to generate the JSIM net list automatically from the schematic view using the circuit view library, and are now developing the filter for that. The circuit simulation results of a 1-bit full adder are shown in Fig. 7.

Table I shows a comparison of simulation results of 1-bit full adder performed by Verilog-XL and JSIM. The delay_Carry and a delay_Sum represent the propagation delay of the carry output and the sum output, respectively, measured from the carry input. One can observe that both simulation results coincide fairly well within an error of $3 \%$. Small discrepancies are caused by the small interaction between the basic cells, which affect the propagation delay of the whole circuit. Note that the simulation time is considerably decreased by using the logic simulation: $270 \mathrm{~s}$ for JSIM and $0.7 \mathrm{~s}$ for Verilog-XL using the SUN Uitra 1.

\section{E. Layout Extraction}

The last step is a layout extraction from the schematic view by using the layout view library. Fig. 8 shows a layout view of the 1-bit full adder. After some other circuit blocks, e.g. $\mathrm{DC} / \mathrm{SFQ}$ converters for input/output circuits, are integrated on the layout editor, a design rule checking and layout vs.

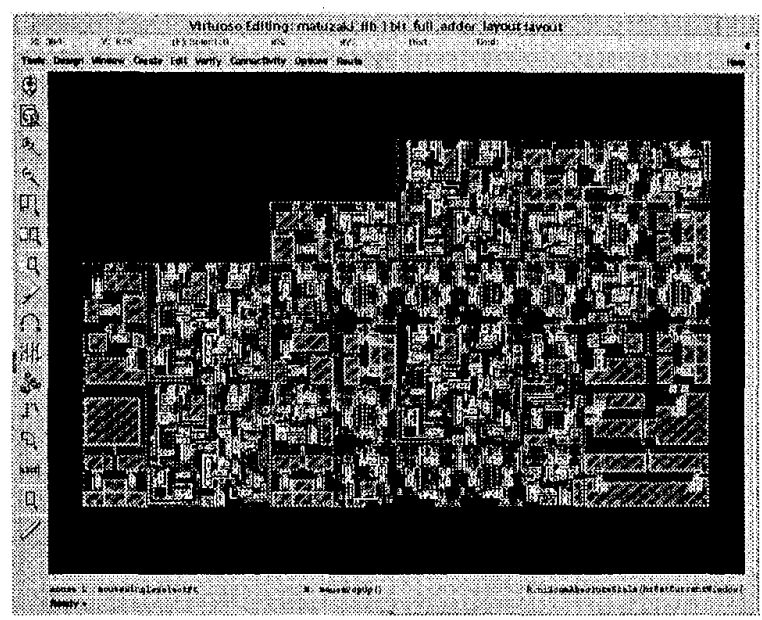

Fig. 8. A layout view of the BDD RSFQ 1-bit full adder.

schematic checking are performed by the Diva to remove careless errors.

\section{CONCLUSIONS}

We have proposed a top-down design methodology for the RSFQ logic circuits based on a binary decision diagram (BDD). We can dramatically reduce the implementation effort and the design time by using a limited number of library cells based on the BDD. The logic level simulation also helps to decrease the design time in the timing optimization.

\section{REFERENCES}

[1] K. K. Likharev and V. K. Semenov, "RSFQ logic/memory family: A new Josephson-junction technology for sub-terahertz-clock frequency digital systems," IEEE Trans. Appl. Superconductivity, vol. 1, pp. 1-28, March, 1991

[2] V. K. Semenov Yu. A. Polyakov, and D. Schneider, "Implementation of oversampling analog-to-digital converter based on RSFQ logic," Extended abstracts of ISEC' 97 , vol. 1, pp. 41-43.

[3] K. Gaj, Q. P. Herr, V. Adler, A. Krasniewski, E. G. Friedman and M. J. Feldman, "Tools for the computer-aided design of multigigahertz superconducting digital circuits," IEEE Trans. Appl. Superconductivity, vol. 9, pp. 18-38, March, 1999.

[4] N. Yoshikawa, H. Tago and K. Yoneyama, "A new design approach for RSFQ logic circuits based on the binary decision diagram," IEEE Trans. Appl. Superconductivity, vol. 9, pp. 3161-3164, March, 1991.

[5] N. Yoshikawa and J. Koshiyama, "A cell-based design approach for RSFQ circuits using binary decision diagram," Supercond. Sci. Technol. vol.12, pp.782-785, 1999.

[6] S. B. Akers, "Binary decision diagram," IEEE Trans. Comput., vol. C-27, pp.509-516, 1978.

[7] T. Kuroda and T. Sakurai, "Overview of Low-Power ULSI Circuit Techniques," IEICE TRANS. ELECTRON., vol. E78-C, pp.334-343, April, 1995.

[8] N. Yoshikawa, Z. J. Deng, S.R. Whiteley and T. Van Duzer, "Data-driven self-timed RSFQ demultiplexer," Applied Superconductivity, vol. 6, pp.361-365, 1998.

[9] K. Gaj, C. H. Cheah, E. G. Friedman and M. J. Feldman, "Functional Modeling of RSFQ Circuits Using Verilog HDL," IEEE Trans. Appl. Superconductivity, vol. 7, pp. 3151-3154, June 1997.

[10] E. S. Fang and T. Van Duzer, "A Josephson integrated circuit simulator (JSIM) for superconductive electronics application," Extended Abstracts of ISEC' 89 , Tokyo, Japan, 1989, pp. 407-410. 